\title{
Power Optimization in Fault-Tolerant Mobile Ad Hoc Networks
}

\author{
Oliviero Riganelli \\ Diaprtimento di Matematica e \\ Informatica, Università di Camerino \\ Camerino I-62032, Italy
}

\author{
Radu Grosu, Samir R. Das, C. R. Ramakrishnan, Scott A. Smolka \\ Department of Computer Science \\ Stony Brook University \\ Stony Brook, NY 11794-4400, USA
}

\begin{abstract}
In this paper, we investigate the transmission-power assignment problem for $k$-connected mobile ad hoc networks (MANETs), the problem of optimizing the lifetime of a MANET at a given degree $k$ of connectivity by minimizing power consumption. Our proposed solution is fully distributed and uses a modelbased transmission power adaptation strategy based on modelpredictive control. Specifically, a stochastic model of the network is used by a state estimator to predict the network's future degree of connectivity and remaining energy. The predicted states are used by an optimizer to derive an optimal transmission power assignment sequence which tracks the desired connectivity level $k$ while minimizing energy consumption. Our experimental results on a simulated wireless sensor network comprising 100 mobile nodes reveals that our localized topology control algorithm provides an almost identical control policy to that of a globalized scheme which is solving a fully observable problem. The difference, of course, is in the scalability of our localized solution, which requires much less communication bandwidth and energy than the globalized approach.
\end{abstract}

\section{INTRODUCTION}

Various civil and military applications possess an inherent need for the rapid deployment of mobile users and concomitant network support. Examples include establishing survivable, efficient, dynamic communication for emergency and rescue operations and disaster relief efforts. Centralized and organized network connectivity is inappropriate for such applications; rather, they require mobile ad hoc networks (MANETs) [1]. For this reason, and due to the fact that such networks are emblematic of the purest form of distributed systems, MANETs have in recent years have become a topic of intense interest within the networking research community.

A MANET is an autonomous collection of mobile devices that communicate over wireless links. Mobile devices are typically powered by batteries, and it is expensive and sometimes infeasible to recharge them. MANETs are intrinsically decentralized, meaning that all network activities, including discovering the topology, must be executed by the nodes themselves. The absence of a centralized infrastructure makes these networks an attractive solution for implementing and managing mobile wireless sensor networks. Wireless sensor networks [2] are an important emerging technology for monitoring the physical world via a large number of distributed sensing node. Many applications [3] [4] [5] [6] need sensor nodes to be mobile making such networks similar to MANETS. However, sensor networks are typically more resource con- strained compared to MANETS because of the use of small, embedded devices and impracticality of recharging batteries. Thus, mobile sensor networks make a challenging case study in our context.

Complicating matters further is the fact that, in these networks, hosts are also routers and this poses a major robustness problem. Nodes running out of battery power not only lose their own individual capabilities, but also impact the entire network by changing routing functionality. In addition, connectivity is strongly influenced by frequently changes in topology due to node mobility.

In the paper, we present a novel, power-aware approach for increasing the robustness of MANETs. The robustness of a wireless ad hoc network is characterized by its level of $k$ connectivity over time. A $k$-connected network has $k$ disjoint connecting paths between any pair of nodes. The particular problem we are interested in solving is the transmission-power assignment problem for $k$-connectivity (TPAP): optimizing the lifetime of a MANET at a given degree $k$ of connectivity by minimizing power consumption. Algorithms that address this problem are sometimes referred to as topology control algorithms, and this shall be the case here. The interest in studying the TPAP for $k$-connected MANET is motivated by the fact that, when a network is $k$-connected, up to $k-1$ node failures can be tolerated without disconnecting the network.

We approach this optimization problem from a decisiontheoretical point of view. A stochastic model of the network is used by a state estimator to predict the network's future connectivity degree and remaining energy. The predicted states are used by an optimizer to derive an optimal transmission power assignment sequence that steers the network behavior to the desired connectivity level $k$ while minimizing energy consumption. Our topology control algorithm is fully distributed, with each node utilizing $p$-hop neighborhood link information in order to estimate global network connectivity.

In order to evaluate our algorithm's performance, we developed a comprehensive simulation model of a wireless sensor network comprising 100 mobile nodes using the Castalia opensource simulation environment [7]. Our experimental results reveal that our localized topology control algorithm, which is solving a partially observable problem, provides an almost identical control policy to that of a globalized scheme, which is solving a fully observable problem. The difference, of course, 
is in the scalability of our localized solution, which requires much less communication bandwidth and energy than the globalized approach.

The rest of this paper develops along the following lines. Section II considers related work. Section III describes our topology control algorithm for the $k$-connected TPAP. Section IV contains our performance-evaluation results, while Section V offers our concluding remarks and future work.

\section{RELATED WORK}

The recent literature describes a number of efforts aimed at increasing the fault tolerance of MANET topologies and devising algorithms for building such topologies. In geometric random graphs, Penrose studied in general the problem of $k$ connectivity of fixed radius networks in a $d$-dimensional cube deployment region [8]. He showed that the communication graph becomes $k$-connected as soon as its minimum node degree reaches $k$ if the number of the nodes goes to infinity and the deployment region is a $d$-dimensional cube. Relatedly, several researchers have studied the problem of kconnectivity in dense ad hoc networks, revealing an interesting analogy with non-geometric random graphs [9] [10]. Although interesting, the theory of geometric random graph cannot be applied in realistic scenarios, because this can be used only to derive results concerning dense ad hoc networks.

In the past years, several topology control algorithms have been also proposed based on different available topology information on each network node. Li and Hou [11] provide a position-based topology control algorithm preserving $k$ connectivity; this is position-based topology control algorithm preserving $k$-connectivity. Their algorithm is position-based because it uses information about node locations. about the node location. Two approaches are introduced: one centralized (FGSS) and the other distributed and localized (FLSS). These approaches proposed greedy algorithms in the sense that at each iteration, the edge with minimum weight is chosen until the $k$-connectivity is satisfied. In this kind of approach, the nodes are usually equipped with a low-power GPS receiver for providing node location estimation, which decreases message exchange among nodes. The CBTC topology control protocol of [12] is direction-based in the sense that relies on the ability of nodes to estimate the relative direction of their neighbors. The key idea is that a node must retain connections to at least one neighbor in every direction defined by a specific parameter $\rho$. Bahramgiri et al. [13] extended CBTC to provide $k$ connectivity. The CBTC protocol is usually implemented using directional antennas for obtaining directional information of the signal sources.

These approaches, unlike our own, do not deal with node mobility because they construct a $k$-connected topology in stationary networks. Considerable work has been reported in the literature for stationary networks and few efforts have focused on the issue of topology control with mobility. The dynamic nature of mobile networks requires periodical information exchange to reorganize the topology, which directly impacts performance of the topology control algorithms.
In mobile scenarios, an interesting topology control scheme for mobile networks is proposed by Zavlanos and Pappas [14], who model connectivity as an imaginary obstacle in a free space and use artificial potential fields to avoid collisions with it. This work, however, focuses more the actuation and control than on the power consumption and transmission power assignment problem.

In mobile ad hoc networks, a scalable topology control needs: (i) to use only local neighborhood information to obtain a topology picture avoiding specific hardware request; (ii) to be executed frequently in order to account for the new positions of the nodes due to mobility. Therefore, the reduction of control traffic overhead is fundamental when implementing topology control mechanisms in mobile networks, because data transmission is the main critical factor that sacrifices device limited resources like energy.

In recent years, significant effort has been made in research and development of MANET and tremendous advancements have been achieved with respect to decision and control theory. In [15], the localization problem of sensor nodes is formulated as an on-line estimation in a nonlinear dynamic system and the authors propose a Robust Extended Kalman state estimator for solving it. Gupta et al. [16] develop a stochastic sensor selection algorithm that decides how schedule the sensors access to the network to minimize the error covariance adopting a state estimator. From a control prespective, a recent survey on packet loss compensation can be found in [17]. In [18], the authors propose a receding horizon control where multiple vehicles cooperate to arrive at multiple target points while maximizing rewards. Although these work does not address $k$ connectivity and power consumption, its relation to our work seems worthy to be investigated.

\section{DESCRIPTION OF OUR APPROACH}

In this section, we describe the topology-control algorithm we have developed for optimizing the lifetime of a MANET at a given degree $k$ of connectivity. We begin with an overview of our approach, followed by detailed discussions of the algorithm's main modules, including the system identification module, the Kalman state estimator, and the model-based power optimizer.

\section{A. Approach Overview}

Our topology control algorithm uses a transmission-power adaptation scheme based on model-based predictive control [19][20]. Referring to this methodology, our control algorithm utilizes a dynamic model of the network to predict and guide the future network behavior in terms of energy consumption and connectivity level. At each sampling interval, an optimal sequence of transmission power assignments is calculated in such a way to optimize a cost objective function over a future horizon. In this way, we characterize the dynamic network model using an input-output black-box modeling technique, in which the inputs $u(t)$ correspond to transmissionpower assignments and the outputs $y(t)$ are the connectivity and remaining energy levels as shown in Figure 1. 


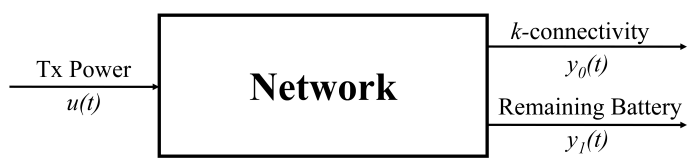

Fig. 1. Dynamic network model.

The proposed topology control algorithm is characterized by the following steps:

1) Initially the future outputs for a determined prediction horizon $P$ are predicted at each sample interval $t$ by the State Estimator using a learned dynamic network model;

2) These outputs $y(t+k \mid t)$ for $k=1, \ldots, P$ depend on the known values up to instant $t$ (past inputs and outputs) and on future inputs $u(t+k), k=0, \ldots, C-1$ where $C$ is the control horizon;

3) The sequence of future inputs is calculated by the optimizer solving a quadratic programming problem in order to keep the future network outputs as close as possible to the predefined reference trajectory $r(t)$, that represents the desired $k$-connectivity and energy level;

4) The first value of this optimal sequence is injected into the network device;

5) The sampling rate is adjusted if the estimation error is out of a predefined admissible range;

6) In the next sample interval we repeat step 1 using the new measured outputs and moving forward both horizons by one time interval.

Every sampling period, each node sends a broadcast message to discover its neighbors and then collects neighborhood link-state information in order to measure the current observable outputs. Maintenance of global network link information requires significant communication overhead especially when nodes are mobile, and in large-scale ad hoc networks the network delay makes this approach impracticable. Localized solutions are therefore preferred. Using a localized solution, a node makes decisions solely based on the link information available from itself and its $p$-hop neighboring nodes. The aggregation of these local link-states constitutes a partial communication graph that is used by nodes to estimate the global $k$-connectivity degree. Although the communication of neighboring link-state information is affected by network delays, the overall delays is significantly smaller than in the global case and can be easily managed by setting the prediction and control horizons in a such way that:

$$
P-C \gg d_{\max } / \Delta t
$$

where $P$ is the prediction horizon, $C$ is the control horizon, $d_{\max }$ is the maximum delay, and $\Delta t$ is the sampling rate. In this way, the communication delay is not significant for the overall control.

In our approach, we learn a stochastic network model based on measured observable data gathered from several test-beds; see Section III-B. The identified network model is then used by a Kalman estimator in order to predict the network behavior over the prediction horizon; see Section III-C. Assuming that the estimates are available, an optimizer computes the opti- mal sequence of transmission-power assignments by solving a quadratic problem in order to trace the desired network behavior over the control horizon; see Section III-D. Figure 2 shows the overall control methodology.

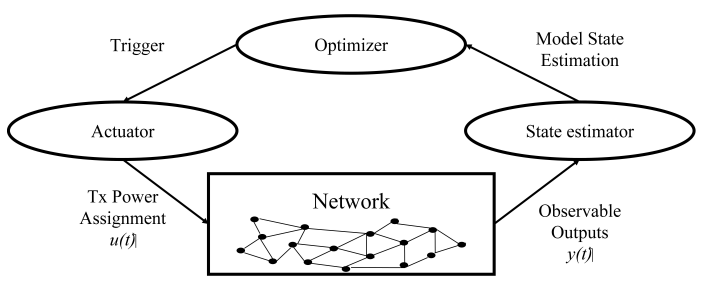

Fig. 2. Overall control methodology for topology control.

In the problem we are solving, we consider mobile symmetric ad hoc networks with omni-directional transmissions. That is, we assume that: $(i)$ the network is dynamic, nodes are mobile, and the topology of the network can change over the time; (ii) all established links are symmetric or bidirectional, so that if a node $u$ is assigned to receive transmissions from a node $v$, then it must also be able to transmit to $v$; (iii) omni-directional transmissions are given by antennas that provide a 360-degree transmission pattern covering an area around the node at a given transmission power. Changing the transmission power changes the dimension of coverage area. The network communication graph is given by an undirected graph $G=(V, E)$, where $V$ is the set of mobile nodes and $E$ is the set of undirected links. In general, this graph may be directed, but our symmetric link constraint allows us to remove all unidirectional links.

\section{B. System Identification}

The dynamic network behavior is unknown and we thus apply a black-box approach to learn its model [21]. This is a very flexible mathematical approach that allows one to build models by analyzing the measured data obtained by experimentation. As such, we can derive the model without knowing the rules that govern the network state. The aim is to model the network dynamics in term of transmission power, $k$-connectivity, and energy consumption. A black-box approach consists of analyzing the input-output data relationship to derive the state-space model structure and parameters. The most important phases to building the model are: $(i)$ experiment design and deployment; (ii) experimental data gathering; (iii) data prepossessing in order to remove possible experimental errors that can affect the final model; $(i v)$ the identification of a multivariable linear time-invariant finite-dimensional system model that "best fits" the experimental data; and $(v)$ model validation.

The input-output data used for learning are provided by a series of test beds. The experimental conditions have been designed for including a wide range of frequencies in order to accurately estimate the model parameters. In this way, given input-output data (estimation data), $<u(t), y(t)>: t=1, \ldots, T$, and a parameterized state space model structure, we estimate the model using standard fitting techniques. Moreover, statistical model validation is performed 
by the residual analysis using a fresh data set, the validation data, for cross validation [22]. Ultimately, we obtain a statespace model in which $x(t)$ is the internal network state, the input action $u(t)$ represents the transmission-power assignment (input action), output $y_{0}(t)$ is the $k$-connectivity level, and output $y_{1}(t)$ is the remaining battery level.

\section{State Estimator}

In the black-box approach, the network state $x(t)$ is not directly measurable. The issue then is to estimate $x(t)$ given access only to the measured outputs $y(t)$ as shown in Figure 3.

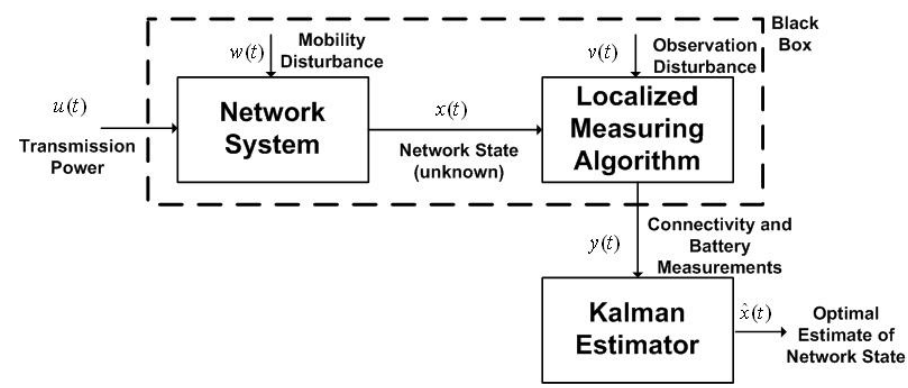

Fig. 3. Kalman Estimator schema used by the topology control algorithm.

The Kalman estimator is an efficient recursive filter that estimates the state of a dynamic system from a series of incomplete and noisy measurements. The estimator is given by a set of recursive equations that provides an efficient computational means to estimate the state of a process in a way that minimizes the mean of the squared error (i.e. the mean square disagreement between the observed value and the estimated value). The estimator is recursive in the sense that it does not need to store all previous measurements and reprocess all data each sampling interval. Rather, the estimator is based on previously learned state space model:

$$
\begin{aligned}
x(t+1) & =A x(t)+B u(t)+w(t) \\
y(t) & =C x(t)+v(t)
\end{aligned}
$$

For zero-initial conditions and in the absence of disturbances one can derive the following truncated step response model [20]:

$$
y(t)=\sum_{i=1}^{n-1} H_{i} \Delta u(t-i)+H_{n} u(t-n) .
$$

where $n$ is the truncation order and $\Delta u(t)=u(t)-u(t-1)$. In (2) and (3), w(t) and $v(t)$ are random variables respectively representing the node mobility disturbance in the state equation and the error sources in the observation equation due to the use of a localized algorithm for the vertex connectivity feedback mechanism. The localized algorithm makes our system partially observable because we estimate a global network property from local link-state information. As such, the model we deal with is a partially observable stochastic model [23]. The random variables are assumed to be independent, white, and, with normal probability distributions

$$
\begin{aligned}
p(w(t)) & \sim N(0, Q(t)), \\
p(v(t)) & \sim N(0, R(t)),
\end{aligned}
$$

where $Q$ and $R$ are the mobility disturbance covariance matrix and the observation disturbance covariance matrix, respectively.

The Kalman Estimator estimates the network state using a form of feedback control: the estimator estimates the state at time $t$ and then obtains feedback by $k$-connectivity and battery measurements. As such, the equations for the Kalman estimator fall into two groups: time-update equations and measurement-update equations. See Figure 4.

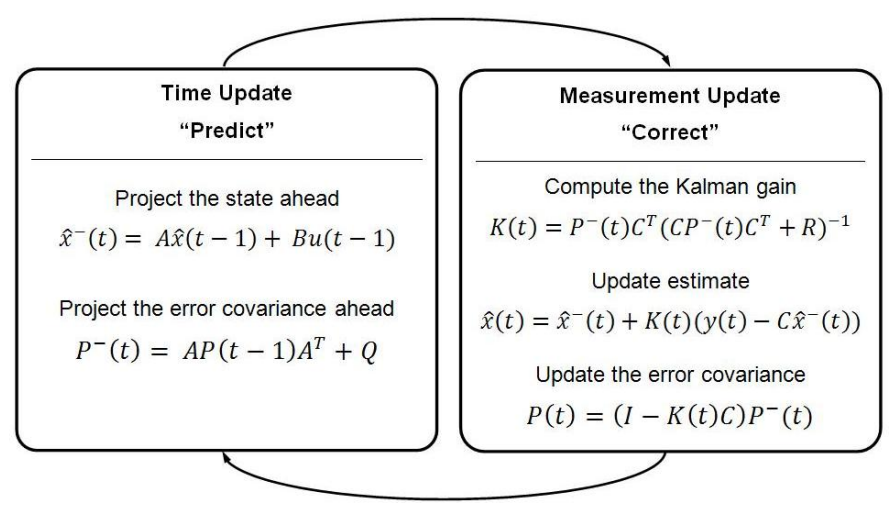

Fig. 4. Kalman Estimator cycle.

The time-update equations are responsible for projecting forward (in time) the current state and error covariance estimates $\hat{x}(t-1)$ and $P(t-1)$, respectively, to obtain the a priori estimates $\hat{x}(t)^{-}$and $P(t)^{-}$for the next time step. As new observations are obtained, the measurement-update equations are responsible for incorporating these observations into the a priori estimate to obtain a more accurate posteriori estimate $\hat{x}(t)$ and to refine the prediction by computing the Kalman gain $K(t)$ which minimizes the posteriori error covariance $P(t)$.

1) Localized k-vertex connectivity algorithm: The distributed algorithm that we have developed for topology control uses only local link-state information to calculate $k$-vertex connectivity, a localized notion of $k$-connectivity. Logically, nodes can make mistakes in their estimate of $k$-connectivity, as it is impossible for them to know about alternate connections in different parts of the network. However, in many applications, the fact that the whole network is or is not globally $k$-connected may be of limited importance, thereby furthering the appeal of a localized computation. Moreover, localized algorithms incur significantly less computation and communication overhead. In our localized algorithm, each node $u$ utilizes the connectivity information embedded in the $p$-hop subgraph centered at $u$ (derived from $u$ 's $p$-hop neighborhood) in order to compute $k$-vertex connectivity.

Formally, let $V$ be a set of nodes deployed in a certain bounded two-dimensional region $R$, with $|V|=n$. Given any $p, 0 \leq p \leq n-1$, the $p$-hop subgraph centered at a node $u$ is 
the bidirectional subgraph induced from those nodes within $p$ hops of $u$. We denote this graph by $G_{p}(u)=\left(V_{p}(u), E_{p}(u)\right)$, where $V_{p}$ is the $p$-hop neighbor set of $u$, including $u$ itself and all nodes within $p$-hops of $u$, and $E_{p}(u)$ is the set of existing links among the nodes in $V_{p}(u)$. Note that the hop distance $d(u, v)$ is less or equal to $p$ for all $v \in V_{p}(u)$.

We also define $B_{p}(u)$, the $p$-hop node set of $u$, which is the set of all nodes that are exactly $p$ hops distant from $u$; $d(u, v)=p, \forall v \in B_{p}(u)$. Thus, $V_{0}(u)=B_{0}(u)=\{u\}$, $V_{p}(u)=V_{p-1}(u) \cup B_{p}(u)$, for $p \geq 1$. Our algorithm for $k$ vertex connectivity enriches the $B_{p}(u)$ subgraph by adding cross edges that connect nodes in $B_{p}(u) \subseteq V_{p}(u)$ with nodes in $B_{p+1}(u)$. The aim of this enrichment process is to take into account outgoing disjoint paths that connect the subgraph $G_{p}(u)$ with the subgraph $G_{p+1}(u)$. This is motivated by the fact that $G_{p}(u)$ can be, for example, 12-connected, but there is only a single outgoing disjoint path; we therefore refine the localized estimate in $G_{p}(u)$ by returning 1-connectivity. Summarizing, node $u$ computes $k$-vertex connectivity in the subgraph $G_{p}(u)$ and then refines its estimation if the number of outgoing disjoint paths is less than this quantity.

2) Adaptable Sampling Period: An important aspect of our topology control algorithm is the rate at which it samples the output variables $y_{0}(t)$ (the $k$-connectivity level) and $y_{1}(t)$ (the remaining battery) of the MANET under observation: the higher the sampling rate, the more accurate the view of the network state. The higher the sampling rate, however, the higher the computation and communication overhead incurred by the control policy, and therefore the greater the drain on the battery energy reserves. We therefore use Kalman estimation error to adaptively adjust the sampling rate. Using the dynamic model representing the network, each node can make predictions of future measurement based on the Kalman estimator prediction/correction paradigm. As such, new observations will be compared to previous predictions and an error value will be calculated on the basis of these comparisons. If the error value exceeds the predefined admissible error range, the sampling rate will be increased; otherwise, it will be decreased.

$$
\begin{array}{rll}
\min _{\Delta u(t), \ldots, \Delta u(t+C-1)} & \sum_{l=1}^{P} \quad\|\hat{y}(t+l \mid t)-r(t+l)\|_{\Gamma_{l}}^{2} \\
& + & \|\Delta u(t+l-1)\|_{B_{l}}^{2} \\
\hat{y}(t+l \mid t)= & \sum_{i=1}^{l} & H_{i} \Delta u(t+l-i) \\
& + & \sum_{i=l+1}^{n-1} H_{i} \Delta u(t+l-i) \\
& + & H_{n} u(t+l-n)+\hat{d}(t+l \mid t) \\
\hat{d}(t+l \mid t)= & \hat{d}(t \mid t) & =y(t)-\sum_{i=1}^{n-1} H_{i} \Delta u(t-i) \\
& +\quad
\end{array}
$$

$$
\begin{aligned}
& \hat{y}(t+l \mid t)=\text { predicted value of } y \text { at time } t+l \text { based on } \\
& \text { the information available at time } t \\
& \hat{d}(t+l \mid t)=\text { predicted value of the process disturbances } \\
& \text { output at time } t+l \text { based on information } \\
& \text { available at time } t \\
& H_{i}=\text { model step response matrix coefficient } \\
& n=\text { truncation order } \\
& \|x\|_{Q}^{2}=x^{T} Q x \text {, norm of } x \text { weighted by } Q \\
& B_{l}, \Gamma_{l}=\text { weighting matrices }
\end{aligned}
$$

\section{Optimizer}

Let $\mathrm{P}$ and $\mathrm{C}$ be the prediction and control horizons, respectively. Assuming that the estimates of $x(t)$ are available at time $t$, the optimal setting of the transmission power is obtained by solving the quadratic problem defined in (7). The first term represents the predicted deviation at future instants between the system output and the reference points $r(t)$ and is used to track the desired behavior. The second term guides the adjustment in the transmission power assignment. The vector $r(t)$ consists of $r_{0}(t)$, the desired $k$-connectivity level (e.g. 10-connectivity) and $r_{1}(t)$, the desired remaining battery level, which is set to the maximum battery charge since the aim is to save energy. In equation (8), consisting of four terms, the future outputs of the system are predicted. The first includes the present and all the future input actions, which are calculated by solving (7). The second and third terms include the past values of the input action that are known at time $t$. The fourth is the predictive disturbance, which is assumed constant for all future time, but at time $t, \hat{d}$ is estimated as the difference between the measured system output $y(t)$ and the output predicted using the process model. In each sampling period, the quadratic problem is solved resulting in new optimal transmission power assignments.

\section{EXPERIMENTAL RESULTS}

In order to provide an experimental evaluation of the proposed approach, we have implemented our topology control algorithm in the Castalia open-source simulation environment for wireless sensor networks [7], which is in turn built on top of the OMNeT++ simulation environment for MANETs and other forms of communication networks [24]. The choice of Castalia was motivated by the fact that it provides an accurate channel/radio model based on empirically measured data and takes into account usually neglected issues such as clock drift, sensor bias, sensor energy consumption, and CPU energy consumption. Castalia also monitors key computational resources, such as memory usage and CPU time.

Our Castalia-based simulation model captures a wireless sensor network of Mica2 motes using Chipcon CC1100 transceivers. The mobility model is one in which nodes move randomly with obstacle-free trajectories in a two dimensional space. This is a memoryless mobility pattern because it retains no knowledge concerning its past location and speed values. In other words, the current speed and direction of a node have no relation to its past values in the previous epoch. Tables I and II provide the configuration parameters for the simulation and the linear quadratic problem, respectively. 
The wireless channel model is a log-normal shadowing model [25], and is given by:

$$
P L(d)=P L\left(d_{0}\right)+10 n \log _{10}\left(\frac{d}{d_{0}}\right)+X_{\sigma}
$$

where $P L(d)$ is the path loss (power density attenuation) at transmitter-receiver distance $d, d_{0}$ is a reference distance, $n$ is the rate at which the signal decays, and $X_{\sigma}$ is a zeromeans Gaussian random variable with standard deviation $\sigma$ which models the shadowing effect. As such, an irregular radio coverage area can be obtained by setting $\sigma \neq 0$.
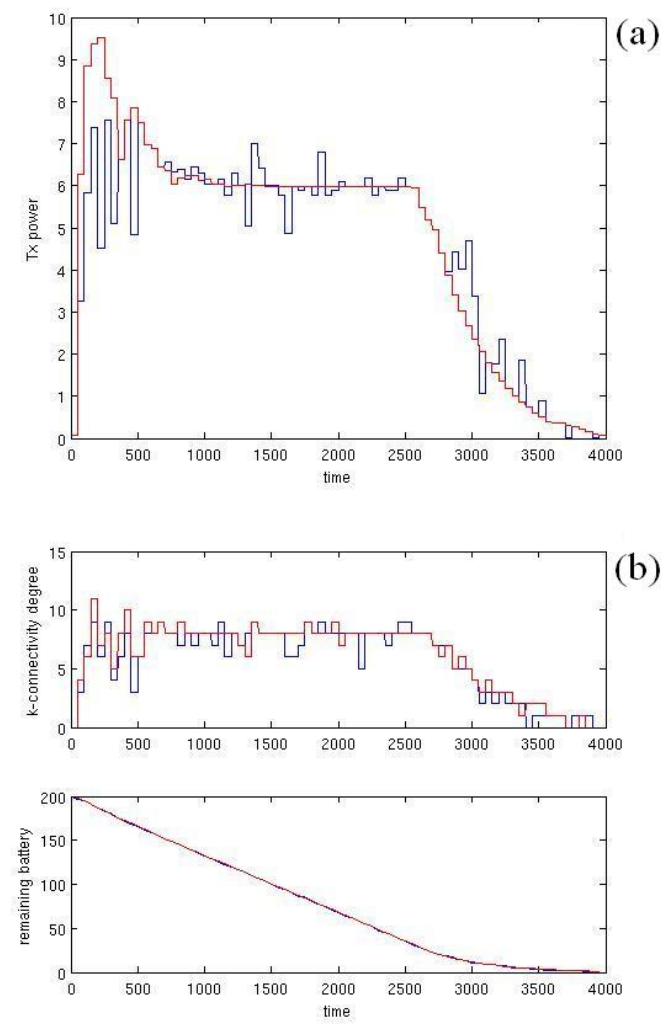

Fig. 5. Comparison of global and local control on node 25 .

For experimental evaluation, a globalized solution, where link state information are acquired by exchange of information, is going to be less competitive due to overhead communication and network delay. For this reason, we report the results of a simulation comparing a globalized solution, where information about neighborhood nodes are available without communication, to our localized solution. In this case, we assume an "external watcher", which can access to neighbors tables of all nodes without requesting communication. In this way, the "external watcher" can build the exact picture of the network and find the optimal policy for the TPAP. The applied localized approach requires only the knowledge of a 5-hop neighborhood. Obviously, the globalized solution without link state information exchange is the best one can achieve because it solve a fully observable decision problem finding the optimal transmission power assignments.
Figures 5(a) and 6(a) compare the transmission power assignments of the globalized (red line) and localized (blue line) algorithms for nodes 25 and 57, respectively. Likewise, Figures 5(b) and 6(b) compare the $k$-connectivity and batterylevel measurements of the global (red line) and local (red line) approaches. The comparison reveals that our localized topology control algorithm, which is solving a partially observable problem, provides an almost identical control policy to that of the globalized scheme. The difference, of course, is in the scalability of our localized solution, which requires much less communication overhead and energy than an implementable globalized one that needs link state information exchange.
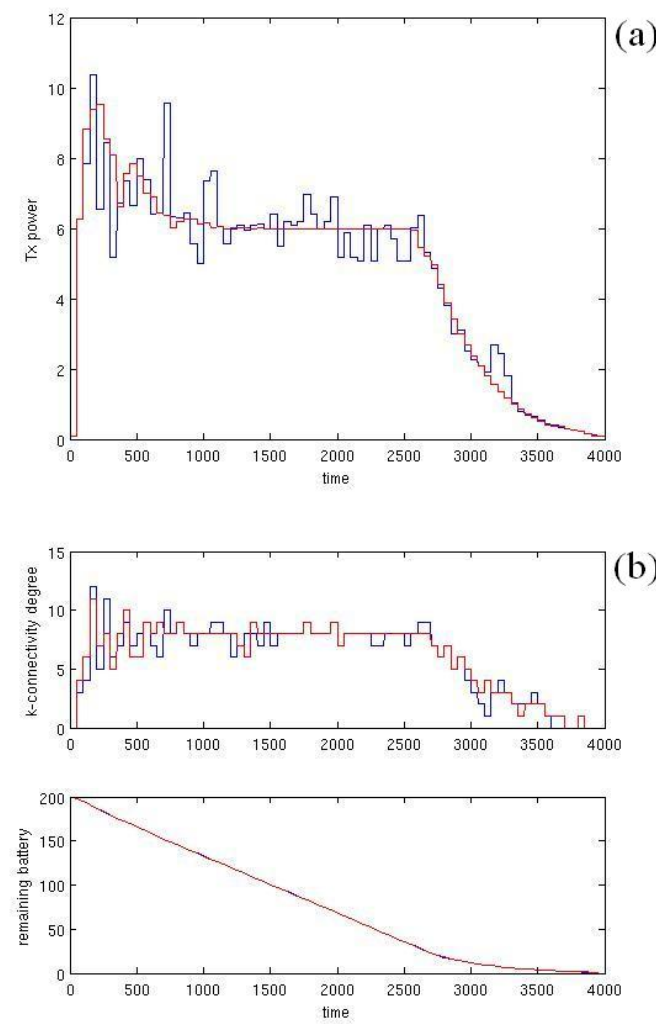

Fig. 6. Comparison of global and local control on node 57.

\begin{tabular}{|c|c|}
\hline Simulation Area & $50 \times 50 \mathrm{~m}$ \\
\hline Nodes & 100 \\
\hline Start Battery & $200 \mathrm{~J}$ \\
\hline Simulation Time & $4000 \mathrm{sec}$ \\
\hline
\end{tabular}

TABLE I

SimULATION SETTING

\section{CONCLusions}

Topology control is one of the most important techniques used in mobile ad hoc networks to reduce energy consumption while concomitantly satisfying global network properties. In this paper, we discussed the importance of scalable topology control for improving fault tolerance in MANETs. We 


\begin{tabular}{|c|c|c|}
\hline \multirow{2}{*}{ Reference Points } & $r_{0}$ & 200 \\
& $r_{1}$ & 8 \\
\hline \multirow{2}{*}{ Output Weights } & $\Gamma_{0}$ & 1 \\
& $\Gamma_{1}$ & 100 \\
\hline Input Weights & $B$ & 0.8 \\
\hline
\end{tabular}

TABLE II

LINEAR QUADRATIC PROBLEM SETTING

addressed this issue by applying a novel decision-theoretic approach that seeks to optimize the lifetime of a MANET at a given degree $k$ of connectivity. Our proposed solution is fully distributed (each node only needs to know its $p$-hop neighborhood), and uses model-based prediction to minimize communication overhead. An experimental evaluation reveals that our localized topology control algorithm provides an almost identical control policy to that of a centralized scheme which is solving a fully observable problem. The difference is in the scalability of our localized solution, which requires much less communication bandwidth and energy than the centralized one.

\section{REFERENCES}

[1] C. Toh, Ad Hoc Mobile Wireless Networks: Protocols and Systems. Prentice Hall PTR New Jersey, 2002.

[2] I. Akyildiz, W. Su, Y. Sankarasubramaniam, and E. Cayirci, "Wireless sensor networks: a survey," Computer Networks, vol. 38, no. 4, pp. 393422, 2002.

[3] ARGO, "Part of the integrated global observation strategy." [Online]. Available: http://www-argo.ucsd.edu/

[4] P. Juang, H. Oki, Y. Wang, M. Martonosi, L. S. Peh, and D. Rubenstein, "Energy-efficient computing for wildlife tracking: design tradeoffs and early experiences with zebranet," SIGPLAN Not., vol. 37, no. 10, pp. 96-107, 2002.

[5] Glacsweb, “A Sensor Web for Glaciers." [Online]. Available: http://envisense.org/glacsweb/

[6] F. Michahelles, P. Matter, A. Schmidt, and B. Schiele, "Applying wearable sensors to avalanche rescue," Computers \& Graphics, vol. 27, no. 6, pp. 839-847, 2003.

[7] NICTA, "Castalia A simulator for wireless sensor networks." [Online]. Available: http://castalia.npc.nicta.com.au/

[8] M. Penrose, "On k-connectivity for a geometric random graph," Random Structures and Algorithms, vol. 15, no. 2, pp. 145-164, 1999.

[9] C. Bettstetter, "On the minimum node degree and connectivity of a wireless multihop network," in MobiHoc '02. ACM, 2002, pp. 80-91.

[10] P. Wan and C. Yi, "Asymptotic critical transmission radius and critical neighbor number for k-connectivity in wireless ad hoc networks," MobiHoc '04, pp. 1-8, 2004.

[11] N. Li and J. Hou, "FLSS: a fault-tolerant topology control algorithm for wireless networks," MobiCom, pp. 275-286, 2004.

[12] L. Li, J. Halpern, P. Bahl, Y. Wang, and R. Wattenhofer, "A cone-based distributed topology-control algorithm for wireless multi-hop networks," IEEE/ACM TON, vol. 13, no. 1, pp. 147-159, 2005.

[13] M. Bahramgiri, M. Hajiaghayi, and V. Mirrokni, "Fault-Tolerant and 3-Dimensional Distributed Topology Control Algorithms in Wireless Multi-hop Networks," Wireless Networks, vol. 12, no. 2, pp. 179-188, 2006.

[14] M. M. Zavlanos and G. J. Pappas, "Distributed connectivity control of mobile networks," Decision and Control, 2007 46th IEEE Conference on, pp. 3591-3596, 12-14 Dec. 2007.

[15] P. Pathirana, N. Bulusu, A. Savkin, and S. Jha, "Node localization using mobile robots in delay-tolerant sensor networks," Mobile Computing, IEEE Transactions on, vol. 4, no. 3, pp. 285-296, May-June 2005.

[16] V. Gupta, T. H. Chung, B. Hassibi, and R. M. Murray, "On a stochastic sensor selection algorithm with applications in sensor scheduling and dynamic sensor coverage," Automatica, vol. 42, no. 2, pp. 251-260, February 2006.
[17] J. P. Hespanha, P. Naghshtabrizi, and Y. Xu, "A survey of recent results in networked control systems," Proceedings of the IEEE, vol. 95, no. 1, pp. 138-162, 2007.

[18] W. Li and C. Cassandras, "A cooperative receding horizon controller for multivehicle uncertain environments," Automatic Control, IEEE Transactions on, vol. 51, no. 2, pp. 242- 257, 12 Feb. 2006.

[19] E. Camacho and C. Bordons, Model Predictive Control. Springer, 2004.

[20] C. E. Garcia, D. M. Prett, and M. Morari, "Model predictive control: theory and practice a survey," Automatica, vol. 25, no. 3, pp. 335-348, 1989.

[21] L. Ljung, System identification: theory for the user. Prentice-Hall, Inc. Upper Saddle River, NJ, USA, 1986.

[22] A. Pagan and A. Hall, "Diagnostic tests as residual analysis," Econometric Reviews, vol. 2, no. 2, pp. 159-218, 1983.

[23] M. Davis and R. Vinter, Stochastic Modelling and Control, Monographs on Statistics and Applied Probability. Chapman and Hall, London/New York, 1985.

[24] A. Varga et al., "The OMNeT++ discrete event simulation system," ESM 2001, 2001.

[25] M. Zuniga and B. Krishnamachari, "Analyzing the transitional region in low power wireless links," IEEE SECON 2004, pp. 517-526, 2004. 